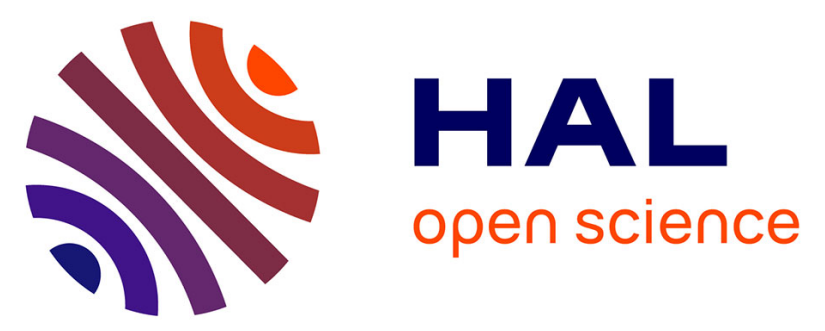

\title{
Infrared spectroscopic measurements of carbon monoxide within a high temperature ablative boundary layer
}

Sean Mcguire, Augustin Tibère-Inglesse, Christophe O Laux

\section{- To cite this version:}

Sean Mcguire, Augustin Tibère-Inglesse, Christophe O Laux. Infrared spectroscopic measurements of carbon monoxide within a high temperature ablative boundary layer. Journal of Physics D: Applied Physics, 2016, 49 (48), pp.485502. 10.1088/0022-3727/49/48/485502 . hal-02494211

\author{
HAL Id: hal-02494211 \\ https://hal.science/hal-02494211
}

Submitted on 28 Feb 2020

HAL is a multi-disciplinary open access archive for the deposit and dissemination of scientific research documents, whether they are published or not. The documents may come from teaching and research institutions in France or abroad, or from public or private research centers.
L'archive ouverte pluridisciplinaire HAL, est destinée au dépôt et à la diffusion de documents scientifiques de niveau recherche, publiés ou non, émanant des établissements d'enseignement et de recherche français ou étrangers, des laboratoires publics ou privés. 


\title{
Infrared spectroscopic measurements of Carbon Monoxide within a high temperature ablative boundary layer
}

\author{
S. D. McGuire, A. C. Tibère-Inglesse and C. O. Laux \\ Laboratoire EM2C, CNRS UPR288, CentraleSupélec, University Paris-Saclay, Grande Voie des \\ Vignes, 92290, Châtenay-Malabry, France \\ E-mail: ${ }^{1}$ sean.mc-guire@centralesupelec.fr
}

\begin{abstract}
Theoretical studies have indicated that the formation of carbon monoxide within a high temperature ablative boundary layer can significantly alter the afterbody radiative heat transfer to the surface of a reentry capsule. This paper represents a first attempt to experimentally measure the concentration of carbon monoxide within the high temperature boundary layer surrounding an ablative material exposed to an atmospheric pressure air plasma. A plasma torch facility was used to produce the high temperature flow and a sample of ASTERM ablative material was inserted into the flow. At the stagnation point, the heat flux to the surface was estimated at $8 \mathrm{MW} / \mathrm{m}^{2}$ and the surface temperature at $2900 \pm 100 \mathrm{~K}$. Both emission and absorption spectroscopy techniques were used to measure the distribution of carbon monoxide within the flow. Emission spectroscopy yielded better signal-to-noise measurements, but the absorption spectroscopy measurements were used to validate emission measurements. In the cases examined, both emission and absorption measurements were consistent and in agreement with one another. Estimates of carbon monoxide temperature and mole fraction were deduced from the spectra taken within the boundary layer upstream of the stagnation point. No carbon monoxide was observed at the stagnation point. These measurements provide a test case for numerical simulations of plasma-ablator interactions.
\end{abstract}

PACS numbers: $34.35 .+\mathrm{a}, 51.70 .+\mathrm{f}, 52.40 . \mathrm{Hf}, 52.75 . \mathrm{Hn}, 82.20 .-\mathrm{w}$ 


\section{Introduction}

The design of reliable and cost effective heat shield material is an important consideration for future space missions. Depending upon the type of mission (lunar, Mars or comet sample return, etc...), the vehicle can reenter Earth's atmosphere at velocities between $10-15 \mathrm{~km} / \mathrm{s}$, within the hypersonic flight envelope.[1] The strong shockwave that forms around the vehicle intensely heats, via strong compression, the air flowing around the spacecraft. A high temperature shock layer forms between the shock wave and vehicle surface, and the temperature of this shock layer can reach 10,000 $15,000 \mathrm{~K}$. The high temperature of the air leads to ionization, molecular dissociation, excitation and intense gas radiation. Carbon based ablative thermal protection systems are mounted on the spacecraft to protect the vehicle from the intense convective and radiative energy transfer. A large research effort is currently directly towards improving modeling capabilities, improving current technology and reducing the mass required for the thermal protection system in order to increase payload size.

There have been a number of researchers looking at the formation of ablative products within a high temperature ablative boundary layer, and the impact that these products have on radiative heat transfer to the vehicle surface. This work typically involves the use of emission spectroscopy to identify particular species, along with other techniques for measuring surface temperature or recession rates, for example. D'Souza et al.[2] looked at the formation of ablative species resulting from the use of an epoxy coating in the X2 expansion facility at the University of Queensland in 2010. Wernitz et al.[3] used a plasma wind tunnel facility to model the atmosphere of Titan and looked at the formation of $\mathrm{N}_{2}, \mathrm{~N}_{2}^{+}$and $\mathrm{C}_{2}$ species within the ablative boundary layer. Additional work has looked at air and nitrogen flows, and the ablative species introduced within the boundary layer and post shock regions.[4, 5, 6, 7, 8] Much of this work has focused on, or identified the presence of, both $\mathrm{C}$ and $\mathrm{CN}$ emission.

Theoretical work has pointed to the importance of carbon monoxide formation within a high temperature ablative boundary layer. Park et al.[9] proposed a model and predicted species mole fractions, including carbon monoxide, along the stagnation streamline. Martin et al.[10] built upon this work and proposed a set of reactions for modeling the formation of ablative species which form in a high enthalpy air flow about a carbon-phenolic ablator. They summarize some of the important reactions that lead to the formation of $\mathrm{CO}$. These include the following:

$$
\begin{aligned}
& C+N O \leftrightarrow C O+N \\
& C+O_{2} \leftrightarrow C O+O \\
& C N+O \leftrightarrow C O+N
\end{aligned}
$$

Johnston and Brandis[11] have recently simulated the entire flow field about a re-entering space capsule, including the interactions between flow and ablator. They focus on the afterbody region of the reentering capsule and compute that large quantities of carbon monoxide form in the afterbody region of the capsule due to forebody ablation. Carbon monoxide mole fractions of $\chi \approx 0.1$ and temperatures around $5000 \mathrm{~K}$ are predicted in the afterbody region under Stardust entry conditions. 
Under these conditions, they also predict that the carbon monoxide, along with other ablative products, can result in a $40 \%$ increase in afterbody radiative heating. Under Mars return conditions, they predict that the effects of ablation products on afterbody radiative heating are even larger.[12]

The goal of this paper is to supply experimental measurements of carbon monoxide concentration, temperature and distribution within a high temperature boundary layer surrounding an ablator. This work builds upon the previous body of work looking at radiation-ablation coupling. It also draws upon previous research looking at spectroscopy of carbon monoxide under similar conditions. For example, Boubert and Vervisch[13] used laser-induced fluorescence measurements in the ultraviolet region to look at relative concentrations of excited state carbon monoxide within a high temperature boundary layer. Cruden et al.[14] used an electric arc shock tube facility at NASA Ames to simulate entry conditions in Mars and Venus atmospheres. They looked at emission from the VUV to the midinfrared and noted the presence of CO fourth positive emission in the VUV. Depraz et al.[15] looked at infrared emission of high temperature $\mathrm{CO}_{2}$ in a microwave discharge. In addition, they also looked at strong $\mathrm{CO}$ emission from the discharge and used the infrared overtone bands $(\Delta \nu=2)$ of $\mathrm{CO}$ to measure the discharge temperature. In our case, the focus is on performing absolute measurements of ground state $\mathrm{CO}$ concentration and temperature in a high temperature ablative boundary layer. A particular advantage of infrared spectroscopy is that it directly yields the concentrations of the electronic ground state.

For our measurements, a $50 \mathrm{~kW}$ plasma torch facility is used to supply a high enthalpy air flow with a free stream temperature of about $6500 \mathrm{~K}$. ASTERM ablative heat shield material was chosen for these experiments. ASTERM is a carbon-phenolic ablative material developed by Airbus Defense and Space.[16] Both NASA and Airbus are developing carbon-phenolic ablative material and, therefore, this choice is representative of a typical reentry mission. Machined cylindrical samples of ASTERM (density, $350 \mathrm{~kg} / \mathrm{m}^{3}$ ) are inserted into the high enthalpy flow produced by the plasma torch. The total heat flux to the material at the stagnation point is estimated to be about $8 \mathrm{MW} / \mathrm{m}^{2}$ and the material surface temperature reaches about $2900 \pm 100 \mathrm{~K}$. An infrared absorption and emission spectroscopy setup is then used to estimate the concentration, temperature and distribution of the carbon monoxide produced within the boundary layer between the high enthalpy flow and the ablative sample material.

\section{Experimental Setup}

The plasma torch facility used is a TAFA Model 66 inductively coupled plasma (ICP) torch powered by a $120 \mathrm{kVA}$ radio frequency LEPEL Model T-50-3 power supply. The power supply operates at $4 \mathrm{MHz}$ and can supply a maximum of $12 \mathrm{kV} \mathrm{DC}$ and $7.5 \mathrm{~A}$ to the oscillator plates. Details of the plasma torch facility are provided in previous publications: for example, see MacDonald et al.[17, 18, 7] For the experiments presented here, a 2-cm diameter exit nozzle was used. The characteristics of the plasma produced by the torch facility with this $2 \mathrm{~cm}$ exit nozzle have been previously studied and documented in Ref. [17]. In particular, the centerline temperature and velocity are approximately $6500 \mathrm{~K}$ and $140 \mathrm{~m} / \mathrm{s}$, respectively. Figure 1 shows a temperature profile of the plasma, measured without a sample placed in the plasma and taken 1.5 


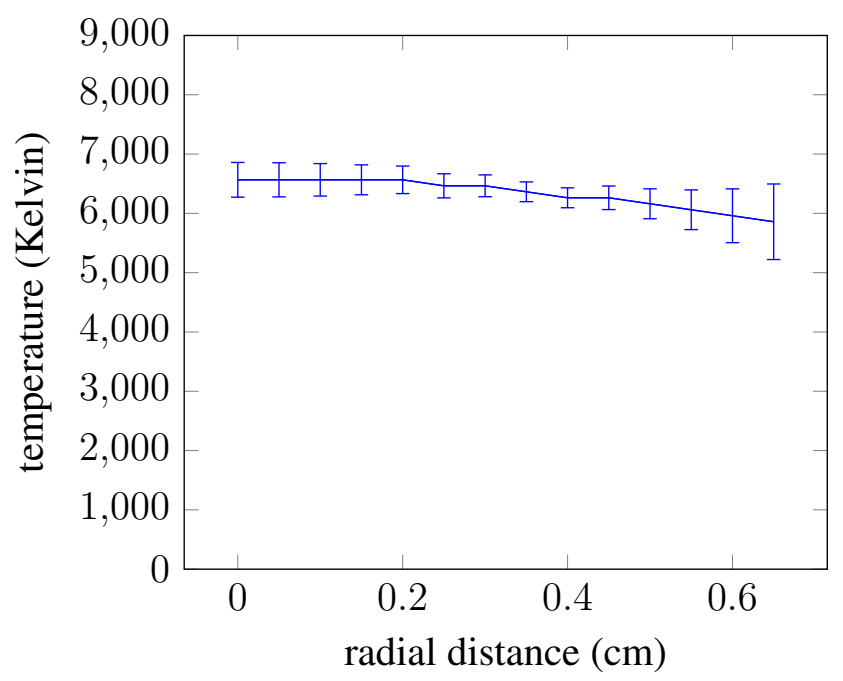

Figure 1. Plasma temperature profile measured $1.5 \mathrm{~cm}$ above nozzle exit from the absolute intensity of the atomic oxygen triplet at $777 \mathrm{~nm} . \mathrm{r}=0$ corresponds to the centerline of the flow.

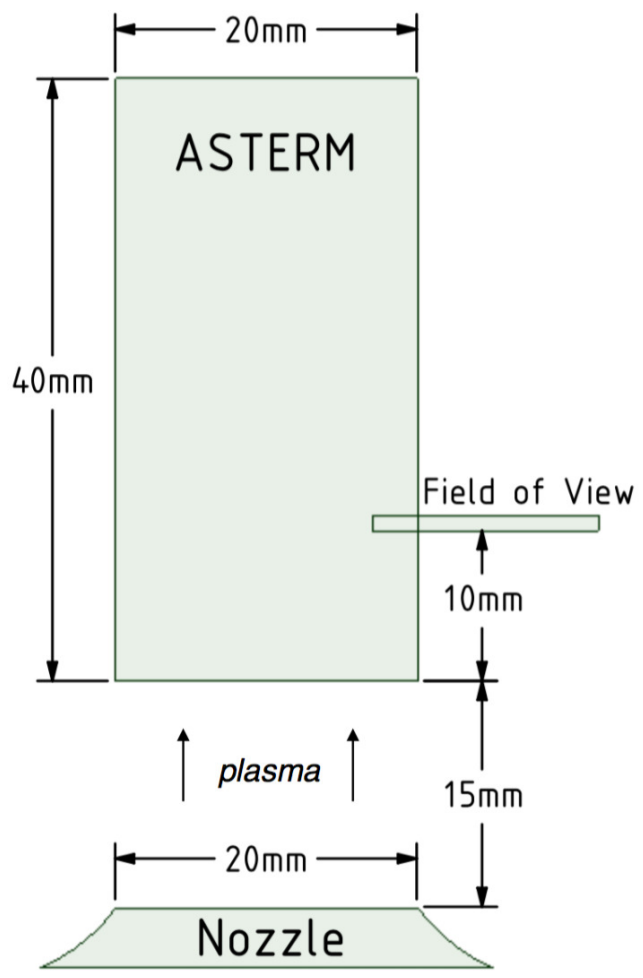

Figure 2. Diagram illustrating the spectrometer field of view, denoted by the rectangle. The field of view is placed partly off sample for imaging of the boundary layer. The field of view is about 15 mm long.

$\mathrm{cm}$ downstream of the nozzle exit. The temperature was measured from the absolute emission of the atomic oxygen line at $777 \mathrm{~nm}$ and the procedure is documented in Ref. [19, 20]. During testing, 
the cylindrical sample of ASTERM ablative test material was inserted into the high enthalpy flow. The sample had a diameter of $2 \mathrm{~cm}$ and a height of $4 \mathrm{~cm}$. Figure 2 details the placement of the ASTERM sample. Figure 3 is an image taken under comparable conditions showing the ablative test sample in the air plasma flow. The surface temperature at the stagnation point was measured using a pyrometer (Minolta Cyclops 153A) and, assuming an ASTERM emissivity of 0.8 based on Ref. [7], is approximately $2900 \pm 100 \mathrm{~K}$.

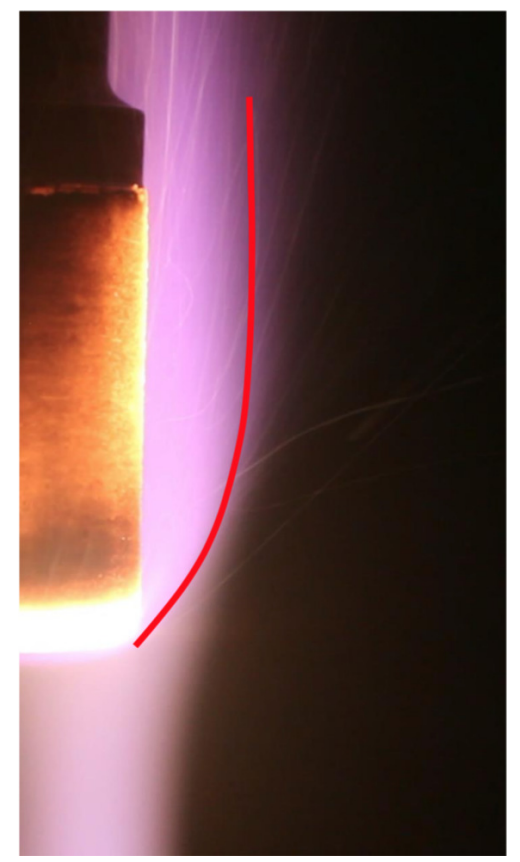

Figure 3. Image showing an ASTERM sample placed above the torch nozzle exit: the flow centerline is on the left boundary of the image. The radiating boundary layer is clearly visible along the sample edge and is between 0.5 and $1 \mathrm{~cm}$ thick downstream of the stagnation point. The purple appearance if probably due to $\mathrm{CN}$ violet emission as observed by MacDonald et al.[7] Note that spallation particles, which are visible in this image, do not interfere with the measurement of carbon monoxide. Grey body emission from these particles represents a continuum and can be distinguished from the carbon monoxide spectral features. In addition, though strong grey body emission from the sample surface was observed, strong continuum emission was not observed within the boundary layer at the wavelengths examined.

An outline of the experimental setup is shown in Fig. 4. This setup permits absorption and emission measurements to be performed simultaneously. All lenses are made of $\mathrm{CaF}_{2}$ and all mirrors are gold-coated. A globar is used as a source for infrared absorption measurements. The light from the globar is collected and focused through a signal chopper before being recollimated and then focused down to the test region. This light, as well as emission from the test region, is collected and focused onto the spectrometer. The signal chopper installed in the optical path enables distinguishing between emission from the test region and light from the globar source using a procedure detailed in Appendix A. The spectrometer is an Acton SpectraPro 2750 equipped with an infrared grating (300 grooves $/ \mathrm{mm}$, blaze $4.0 \mu \mathrm{m})$. An IRC800 camera from IR Cameras is mounted at the exit port for detection of the signal. The camera integration time 


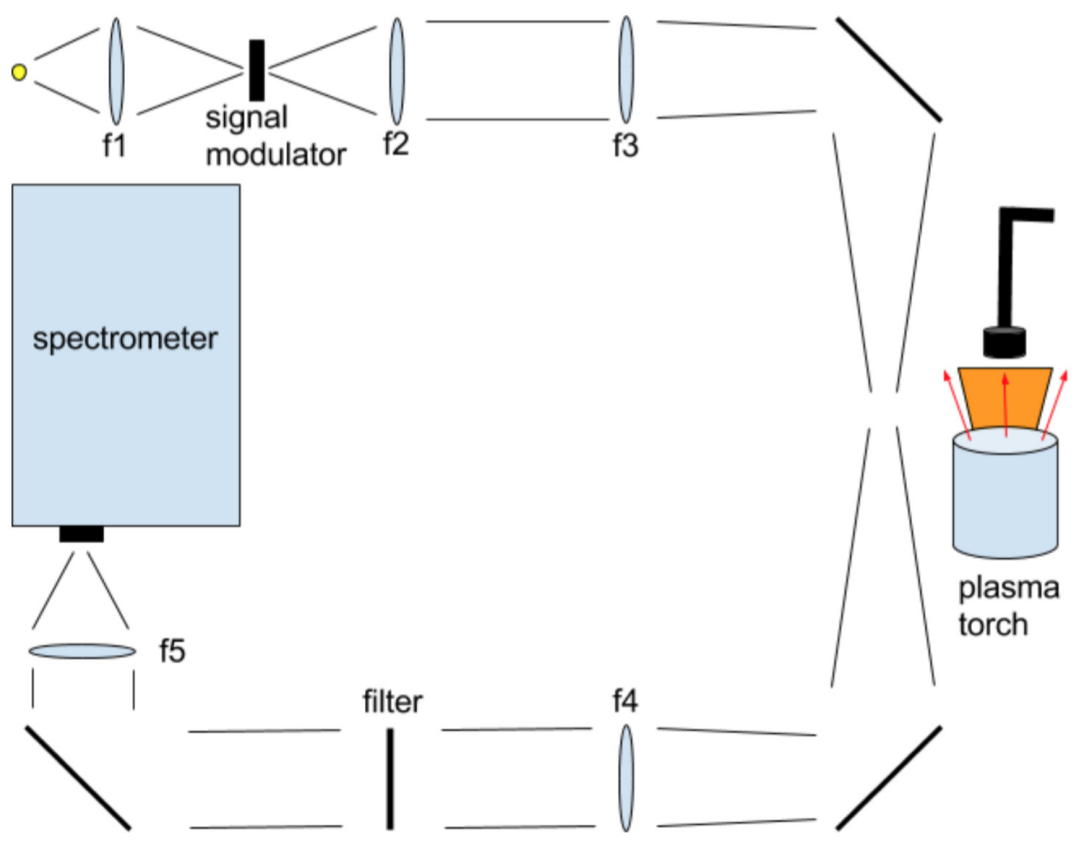

Figure 4. Experimental setup for emission and absorption measurements. The light source is a broadband blackbody. Lenses $\mathrm{f} 1(\mathrm{f}=10 \mathrm{~cm})$ and $\mathrm{f} 2(\mathrm{f}=25 \mathrm{~cm})$ direct the signal through a signal modulator and re-collimate the light. The signal modulator enables distinguishing between emission from the test region and absorption of the globar source. The collimated signal is focused into the test region by $\mathrm{f} 3(\mathrm{f}=50 \mathrm{~cm})$ and re-collimated by $\mathrm{f} 4(\mathrm{f}=50 \mathrm{~cm})$. The final lens $\mathrm{f} 5(\mathrm{f}=25 \mathrm{~cm})$ focuses the signal onto the entrance slit of the spectrometer. The filter is a high pass filter with a cut-off wavelength of $3.6 \mu \mathrm{m}$ and serves to prevent light at $\lambda / 2$ from interfering with measurements at wavelength $\lambda$.

is set to $450 \mu \mathrm{s}$. A single camera image provides spectra over a $30 \mathrm{~nm}$ spectral window. The spectrometer instrumental broadening is measured to be trapezoidal with a base width of $1.7 \mathrm{~nm}$ and a top width of $1.5 \mathrm{~nm}$. The equivalent Full Width at Half Maximum is $1.6 \mathrm{~nm}$. All theoretical spectral simulations were done with the software package SPECAIR[19, 21] using this trapezoidal slit function.

Figure 5 overviews the spectral region selected for measurements. The labeled peaks correspond to spectral features of carbon monoxide from the rotational $\mathrm{R}$ branch and from different vibrational bands. The peak labeled $A$ is the $R(35)$ rotational line from the $(4,3)$ vibrational band. The peak labeled $B$ is a collection of rotational lines: $R(7), R(15)$ and $R(24)$ lines from the $(1,0)$, $(2,1)$ and $(3,2)$ vibrational bands, respectively. The peak labeled $C$ corresponds to the $R(34)$ and $\mathrm{R}(47)$ rotational lines from the $(4,3)$ and $(5,4)$ vibrational bands, respectively. The relative amplitudes of these peaks are sensitive only to temperature over the range of interest.

Upon sample insertion, the ASTERM test sample recedes at a rate of about $10 \mathrm{~mm} / \mathrm{minute}$ over the testing intervals of interest.[17] No emission or absorption signal was observed at $4.6 \mu \mathrm{m}$ before the ASTERM sample was inserted into the plasma. Carbon monoxide signals were only observed after sample insertion, indicating that the carbon monoxide observed was produced only 


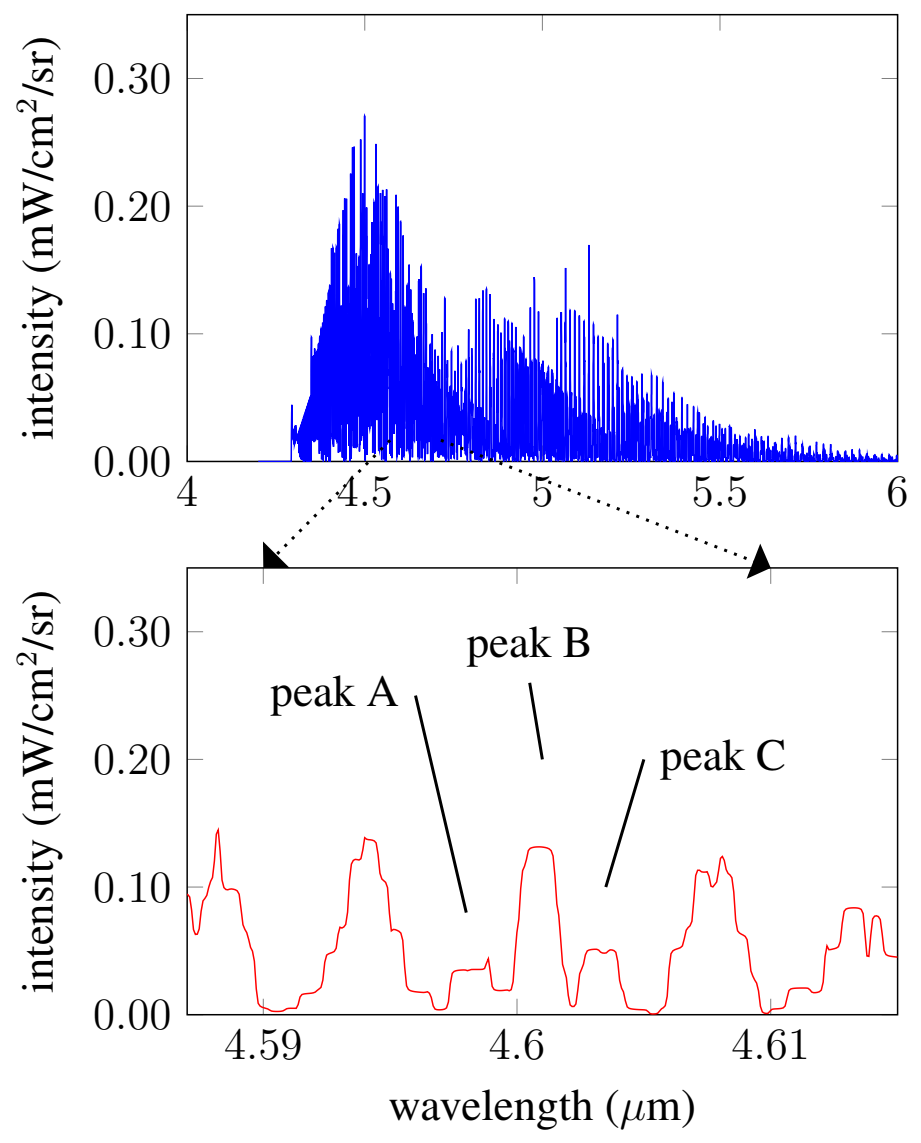

Figure 5. Top: SPECAIR simulations of the emission spectrum of carbon monoxide at a temperature of $4500 \mathrm{~K}$, pressure of $1 \mathrm{~atm}$ and mole fraction of $\chi=0.125$ for an optical path length of $1.4 \mathrm{~cm}$. Bottom: With the spectrometer and camera used for these experiments, a $30 \mathrm{~nm}$ spectral window can be captured in a single image. The spectrum consists of multiple vibrational transitions $\Delta \nu=+1$, each within the rotational R- branch of their respective band group. The features selected here for analysis are free from any room temperature absorption (e.g. from $\mathrm{CO}_{2}$ ) and also strong enough to provide sufficient signal-to-noise ratio. The peaks labeled A, B and C are discussed in the text and used for the temperature and concentration measurements.

from the interaction between the plasma and ASTERM sample. An image sampling frequency of $300 \mathrm{~Hz}$ was used. 500 images were collected and averaged for each batch. There was a one second delay between batches, thus giving a coarse time history of the carbon monoxide concentration and evolution. We present here the batch of images taken about four seconds after sample insertion: early enough that the sample geometry had not changed significantly but late enough that the sample surface temperature had stabilized. For emission measurements, calibration from pixel count to absolute emission intensity was done via a calibrated tungsten lamp (Optronic Laboratories, model OL550).

A relatively strong transient burst of $\mathrm{CO}$ emission is observed within the first two seconds after sample insertion. This transient burst could be at least partially resolved by reducing the images collected per batch, thereby reducing the time necessary to collect the batch and increasing 
the temporal resolution of the measurement. However, within this period, the sample was also observed to vibrate strongly making quantitative analysis difficult. Both the observed transient burst of signal as well as the sample vibrations were observed to settle within the first two seconds after sample insertion, giving way to a steady and stable signal which only varied slowly with time as the sample receded. Measurements reported below were taken during this steady state period. This initial transient burst of signal could be due to pyrolysis of the ASTERM resin: see, for example, the work of Helber et al.[22].

For the batch of images selected for analysis, typical emission signal levels varied between 5 and 20 counts for carbon monoxide emission with an upper bound of about 200 counts for strong continuum surface emission. The emission spectra also ride atop a background of a few thousand counts. A background image was taken for removing dark current, and off-resonance intensity measurements were used to remove continuum emission. Figure 6 shows a sample image taken at the exit of the spectrometer during a test with the setup configured as in Fig. 2. The strong surface emission is easily visible and is seen to be continuum emission as expected. The carbon monoxide emission from the boundary layer can also be seen, though it is much weaker. This image represents a 500-shot average. The spatial dimension corresponds to the horizontal dimension in Fig. 2.

The images used in the processing of absorption spectra are obtained by taking the standard deviation of the batch of images. The full scale intensity, necessary for determining the absorption, is obtained by taking a background image of the globar before insertion of the test sample. Absorbances are then calculated via the Beer-Lambert law. Here, due to the lower signal-to-noise of these measurements, the images were binned across the spatial dimension to produce a spectrum with sufficient signal-to-noise ratio.

\section{Results \& Discussion}

The spatial profile of the central carbon monoxide emission peak (peak B in Fig. 5) and the profile of the background continuum emission (measured off resonance) are shown in Fig. 7. The sample edge is visible in Fig. 7 and is identifiable by the sudden change in both carbon monoxide and continuum emission intensities. There is a strong peak of carbon monoxide emission at the edge of the ablator surface. This peak persisted throughout the entire test, until the ablator surface recessed through the spectrometer field of view and thus indicates that carbon monoxide is being produced directly at the surface via ablation. The carbon monoxide emission extends approximately $6 \mathrm{~mm}$ beyond the sample surface.

In order to have spatially resolved measurements, the spectra were Abel inverted using POLAB4 (Ref. [20]), which is an Abel-inversion program enabling the calculation of propagated

uncertainties. Note that, to provide an accurate representation of the true spatial variation, the flow must be axisymmetric. This is assumed to be true though, because only half-profiles could be collected, the validity of this assumption could not be validated. Only the emission measurements had sufficient signal-to-noise for Abel inversion of the radial profiles. The amplitudes of peaks $\mathrm{A}, \mathrm{B}$ and $\mathrm{C}$ (as identified in Fig. 5) were chosen because the ratio of these peaks is sensitive to 


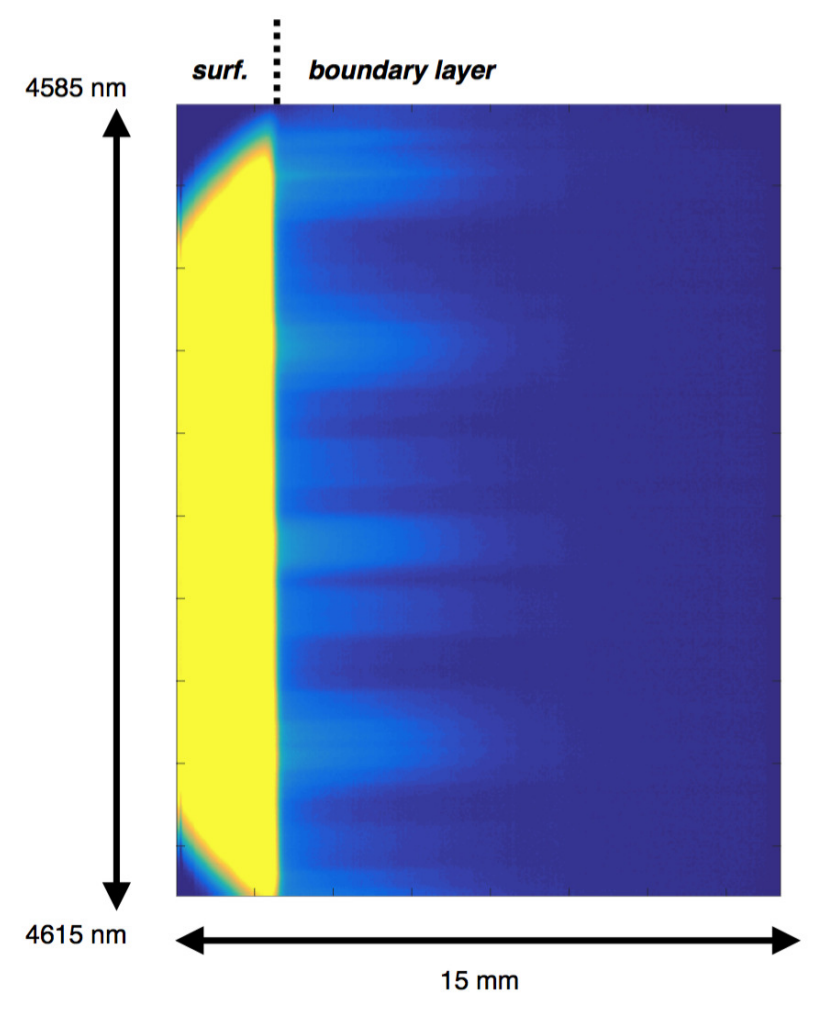

Figure 6. Sample image of emission spectrum at output of spectrometer with the experiment configured as in Fig. 2. The x-direction corresponds to the spatial dimension while the y-direction corresponds to the wavelength dimension. The continuum surface emission is easily seen (yellow). The carbon monoxide emission features are also visible in this image as horizontal streaks which exist within the boundary layer.

temperature over the temperature range of interest. The Abel-inverted intensities of these peaks are shown in Fig. 8. These Abel-inverted peak intensities were used to estimate the temperature and concentration of the carbon monoxide. Figure 9 shows how the peak ratios B/A and B/C vary with temperature as predicted with SPECAIR (Ref. [21]); a peak ratio measured at a particular radius is shown for comparison. Though SPECAIR is capable of simulating a non-equilibrium radiating system, for this work, carbon monoxide was assumed to be in thermodynamic equilibrium so that the full distribution of rovibronic states could be characterized by a single temperature. The structure of the experimental spectrum could then be ascribed to a single temperature and mole fraction. The temperatures estimated from each of the two ratios, $\mathrm{B} / \mathrm{A}$ and $\mathrm{B} / \mathrm{C}$, were found to be consistent with one another across the radial distribution. Because these peaks originate from rotational lines spanning rotational levels $J=7-47$ and vibrational levels $\nu=1-5$, this supports the assumption of equilibrium among the degrees of freedom of carbon monoxide. Once the temperature of the carbon monoxide is known, the absolute magnitude of peak B was used to determine the carbon monoxide concentration.

Figure 10 shows the resulting temperature and concentration profiles. The temperature is $\approx$ $3800 \pm 100 \mathrm{~K}$ near the sample surface $(r=1 \mathrm{~cm})$, and increases steadily to approximately $5700 \pm$ 


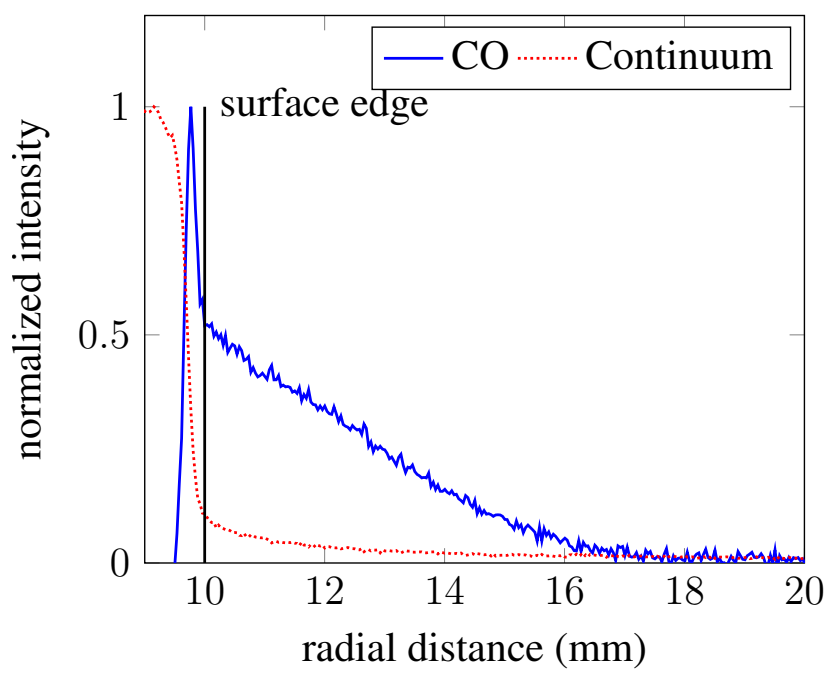

Figure 7. Measured spatial distribution of the carbon monoxide emission signal shortly $(\approx 4 \mathrm{sec})$ after sample insertion. The continuum emission is also shown for comparison. Each signal has been normalized to one so that the relative trends can be observed. In reality, the continuum emission is much stronger than the emission from carbon monoxide.

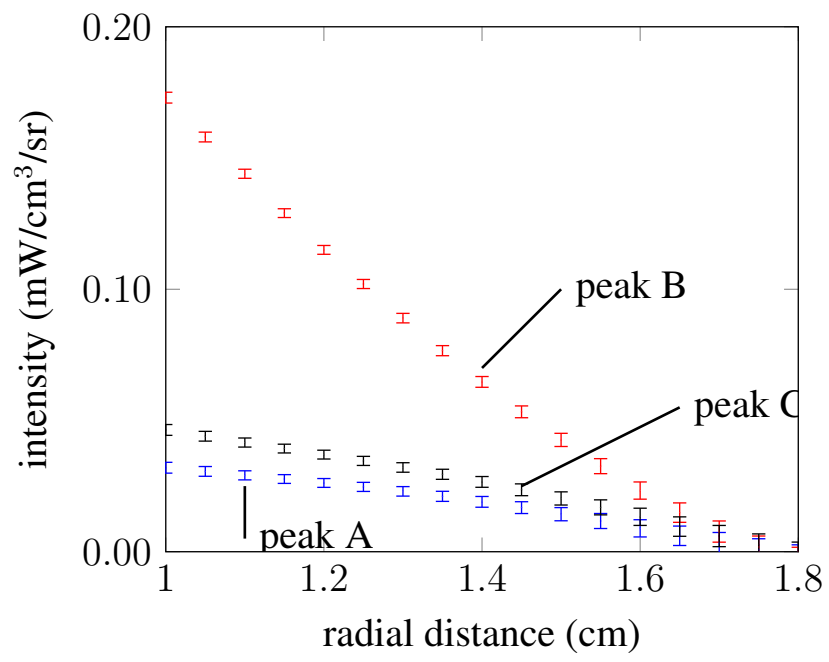

Figure 8. Variation in Abel-inverted peak intensity with radius. The sample surface is located at $r=1 \mathrm{~cm}$.

$1000 \mathrm{~K}$ about $7 \mathrm{~mm}$ away from the sample surface. Note that this is consistent with Fig. 1, where the flow temperature without a sample inserted is estimated to be about $6500 \pm 200 \mathrm{~K}$. The mole fraction of carbon monoxide, on the other hand, starts at about $\chi \approx 0.13$ near the sample surface and decreases steadily, with virtually no carbon monoxide left about $7 \mathrm{~mm}$ away from the sample surface.

For verification of these measurements, both the initial emission and absorption spectra were binned over the entire spatial distribution of Fig. 6. Binning the measured spectra increased the signal-to-noise ratio of the measurements. This was particularly important for the 

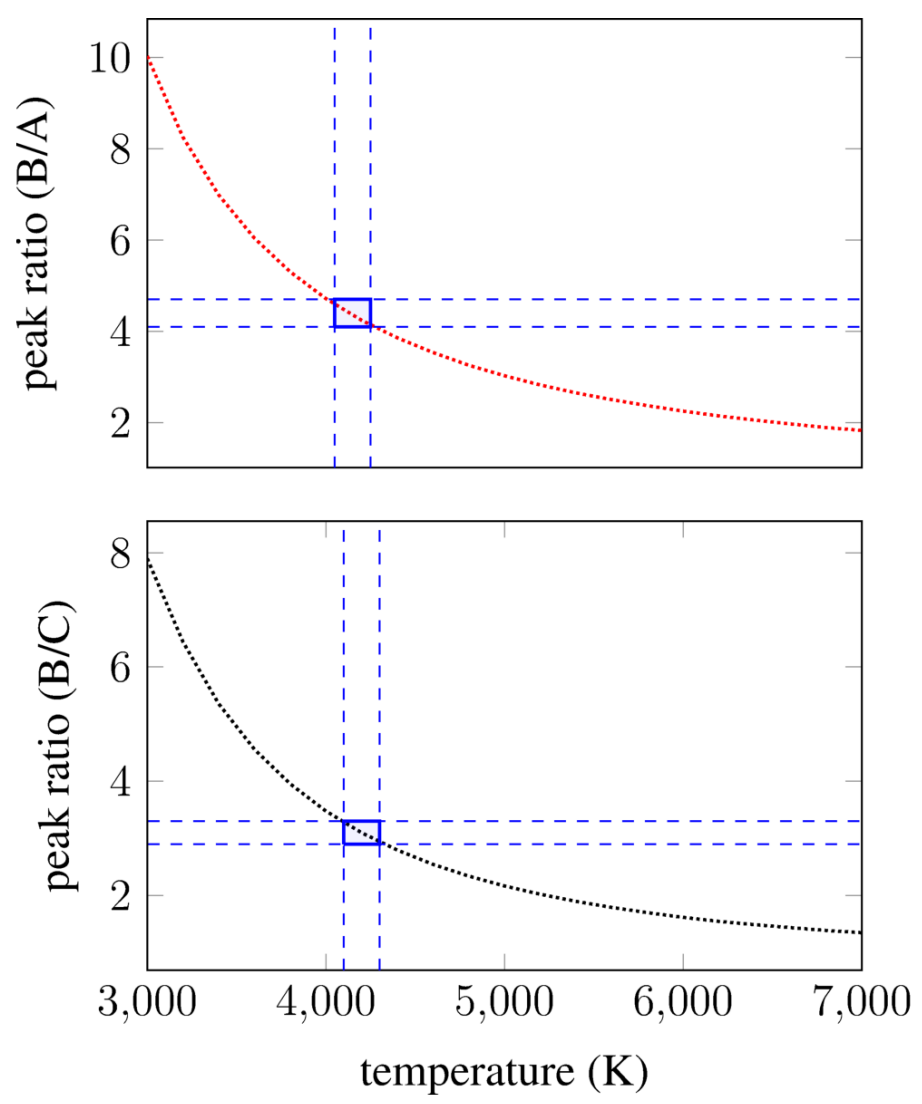

Figure 9. Simulated variation in peak ratio versus temperature. The dashed lines bound the Abelinverted measured peak ratios at a radius of $1.2 \mathrm{~cm}$. Top: Variation of the ratio B/A with temperature. Bottom: Variation of the ratio $\mathrm{B} / \mathrm{C}$ with temperature.

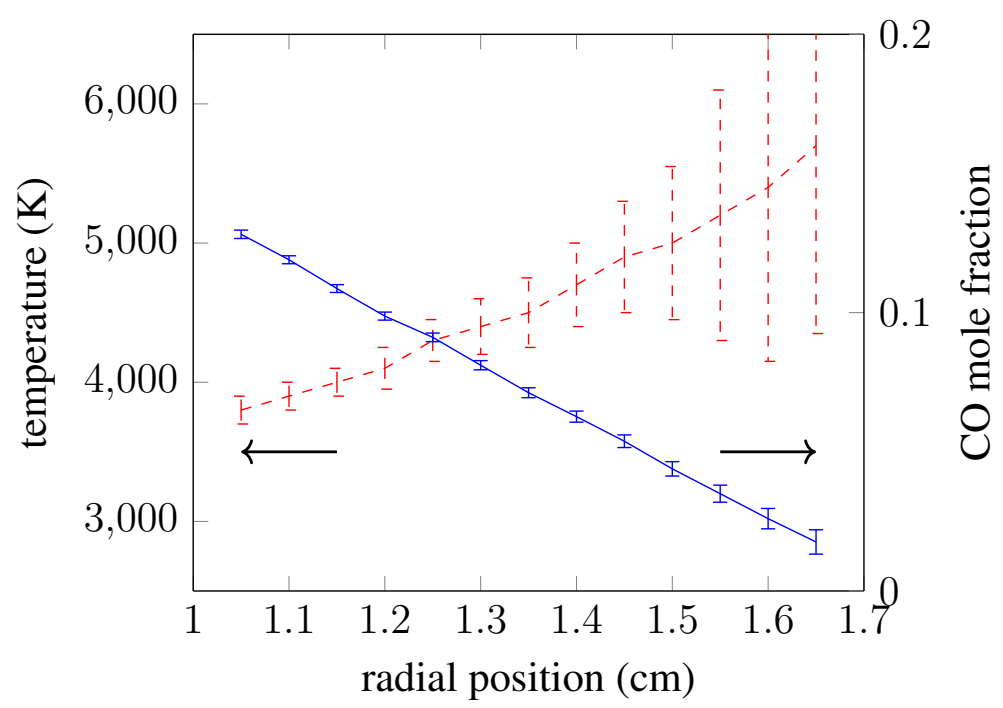

Figure 10. Measured temperature (dashed/red) and CO mole fraction (solid/blue) as a function of radial distance. The sample surface is located at $r=1 \mathrm{~cm}$. 


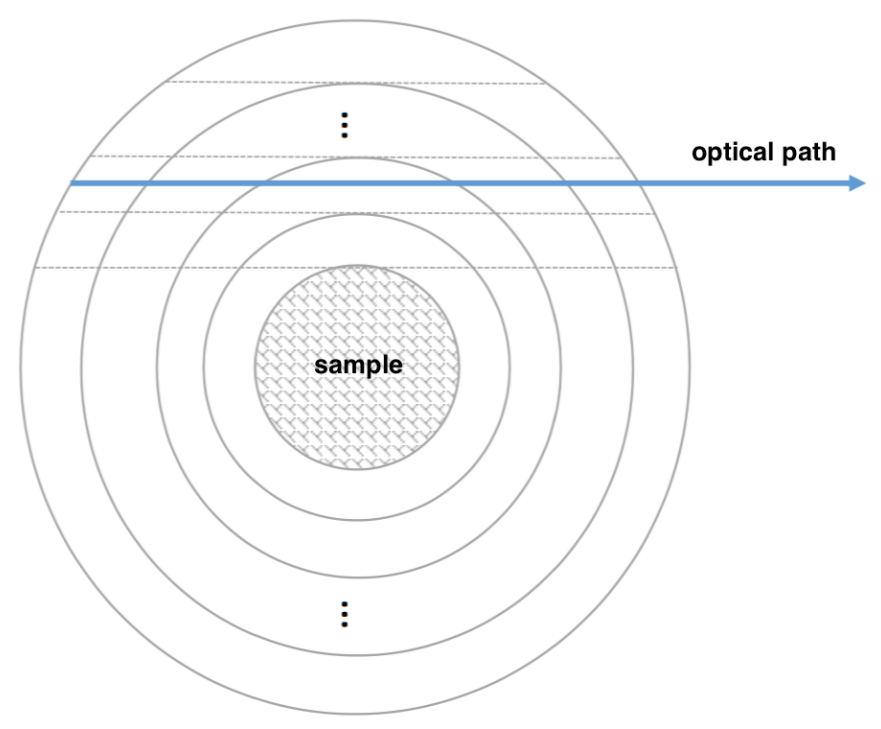

Figure 11. For the emission and absorption slab calculations, the boundary layer is divided into 13 concentric annular slabs. The radiative transport equation is solved on a line-of-sight looking through the boundary layer as indicated, which corresponds to the line-of-sight of the experiment.

absorption measurements, where the signal-to-noise ratio was not sufficient for spatially resolved measurements. The spectra produced in this manner were averaged across a relatively wide range of carbon monoxide temperatures and concentrations. For comparison with these experimental spectra, the measured radial profiles of carbon monoxide temperature and concentration were used to simulate the measured spectra. For this calculation, concentric annular rings were generated, each with the measured carbon monoxide temperature and concentration. Next, the radiative transport equation was solved with SPECAIR along the line-of-sights passing through each ring. Figure 11 illustrates. Finally, these simulated spectra were convolved with the instrumental broadening function and binned across the spatial dimension for comparison with the measured spectra. Figure 12 shows the results. Both emission and absorption spectra are consistent with one another.

The measurements reported were extracted from a batch of images taken approximately four seconds after sample insertion. This choice ensured that the sample geometry had not changed significantly due to ablation. However, we did observe two interesting phenomena after this point. As time progressed, the base of the cylindrical sample, which corresponds to the stagnation point, recessed upward (Fig. 2) and eventually through the spectrometer field of view. We observed that the measured quantity of carbon monoxide continually decreased as the stagnation point recessed towards the field of view. Once the stagnation point entered the field of view, we no longer observed a carbon monoxide signal. Experiments with the field of view located on the center of the sample (radial location, $\mathrm{r}=0$ ) were also conducted and confirmed this observation. We hypothesize that this is due to a difference in temperature between the relatively cold boundary layer and the relatively hot stagnation point. The CEA equilibrium code[23] can be used to calculate the mole fractions of relevant species as a function of temperature, for a particular quantity of carbon 

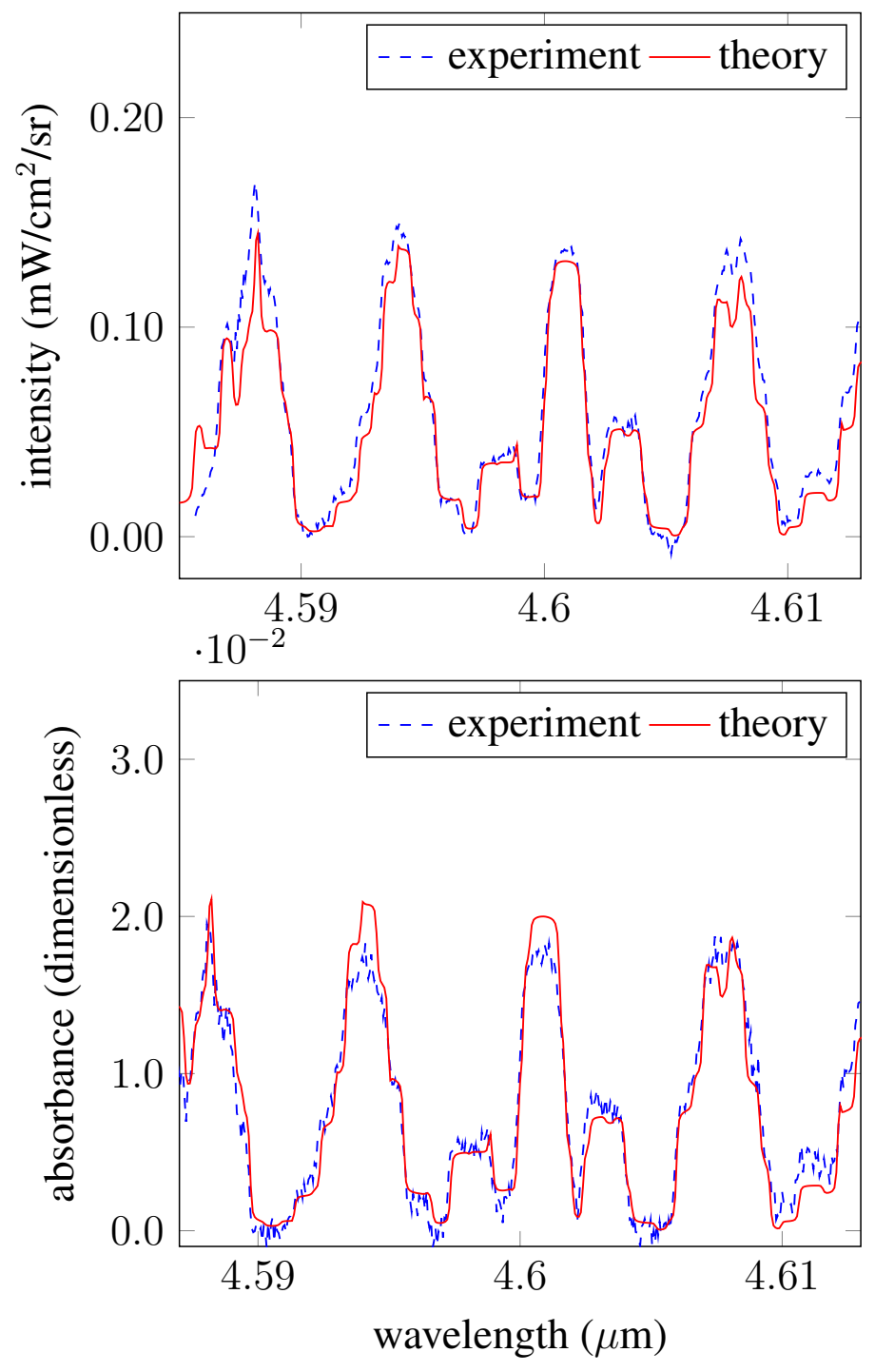

Figure 12. Top: Absolute emission intensity spectra: experimental (after binning) and theoretical spectra for comparison. Bottom: Absolute absorbance spectrum and theoretical spectra for comparison. The discrepancies between experiment and theory here are attributed to the lower signal-to-noise ratio of the absorption signal. The agreement however is quite satisfactory and serves as a good independent validation of the emission measurements.

injected in air. These calculations indicate that, between approximately $3000 \mathrm{~K}$ and $6000 \mathrm{~K}$, carbon monoxide is the dominant species. Above $6000 \mathrm{~K}$, however, the mole fraction of carbon monoxide begins to decrease and that of atomic carbon begins to mount. At about 7000 Kelvin, atomic carbon becomes the dominant species. Given this trend, it is suspected that, near the stagnation point, the elevated temperatures favor the presence of atomic carbon to carbon monoxide. 


\section{Conclusions}

Quantitative infrared emission and absorption measurements of carbon monoxide were made within a high temperature ablative boundary layer. The measurements demonstrate the coupling between ablative surface and high enthalpy flow: carbon monoxide was only observed when the ablative sample was introduced into the high enthalpy flow. Carbon monoxide mole fractions as high as $\chi=0.13$ were measured near the sample surface. This can have potential ramifications for the heat flux to the surface in the afterbody region of a reentering space capsule. Consider, for example, an atmospheric pressure, $2 \mathrm{~mm}$ thick slab of high temperature boundary layer at $4500 \mathrm{~K}$. If ablative species are not accounted for, a simple CEA[23] calculation can be performed to determine the mole fractions of the various species present. SPECAIR may then be used to simulate the emission intensity from the VUV to IR regions of the spectrum This non-ablating case may be compared with an ablating case, where atomic Carbon is added to the CEA calculation such that, at 4500 Kelvin, the equivalent mole fraction of $\mathrm{CO}$ is $\chi_{C O}=0.10$. Fig. 13 shows the result. There is a 300\% increase in the total emission when including ablative species with virtually all of this increase coming from the UV and IR regions of the spectrum: $\approx 2 \mathrm{x}$ increase in the UV via fourth positive emission and $\approx 10 \mathrm{x}$ increase in the IR coming from the infrared bands around 2.3 and $4.6 \mu$. However, in addition to the direct impact of carbon monoxide emission, there are also indirect effects resulting from the presence of the carbon monoxide within the boundary layer. As Johnston and Brandis[11] point out, the presence of ablative species such as carbon monoxide can have an impact on the temperature field in the boundary layer which, in turn, alters the emission and absorption from other species as well. Thus, the situation is not as simple as this calculation would indicate.

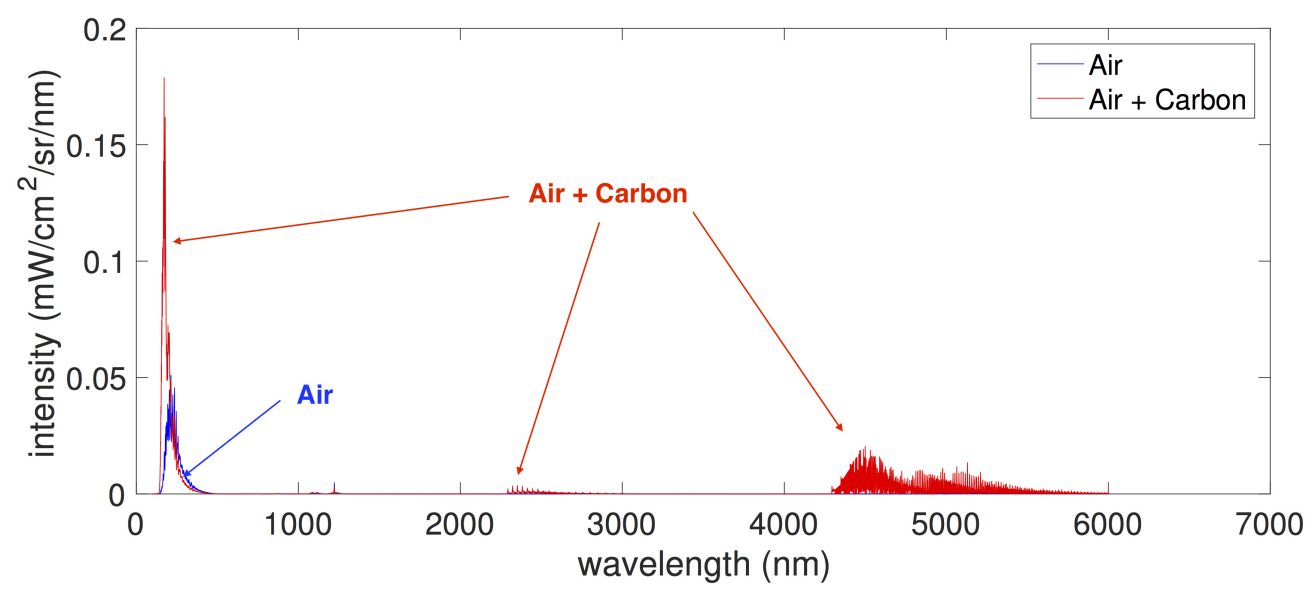

Figure 13. Emission intensity from equilibrium air (blue) at 4500 Kelvin and an Air/Carbon mixture at 4500 Kelvin and $1 \mathrm{~atm}$. All concentrations were calculated using the NASA CEA code. The quantity of carbon was chosen to give a mole fraction of $\chi_{C O}=0.10$ in accordance with the experimental results. The spectra were calculated using SPECAIR.

The measurements presented in this paper provide a test case for numerical simulations 
looking at plasma/ablator interaction. We plan to build upon this work by looking at how the quantity and distribution of carbon monoxide changes when using different sample materials, like graphite. Comparing the results here with those obtained using a material like graphite would help elucidate whether the carbon monoxide is coming from pyrolysis or surface ablation effects. In addition, we plan to use ultraviolet and visible spectroscopy to identify carbon and cyanogen distributions for comparison with the measured carbon monoxide distributions.

\section{Appendix A}

Images were sampled at a frequency $f_{s}$ equal to twice the chopping frequency $f_{c}$. Thus, half of all images include only emission, while the other half include both emission in addition to the light from the globar source. If $I_{p}$ denotes the emission intensity from the plasma and $I_{g}$ denotes the intensity of the globar source, then the following summation represents the average intensity incident on a given pixel across $\mathrm{N}$ samples, where $\mathrm{N}$ is an even number.

$$
\begin{aligned}
\langle I\rangle & =\frac{1}{N}\left[\frac{N}{2}\left(I_{g}+I_{p}\right)+\frac{N}{2}\left(I_{p}\right)\right] \\
& =I_{p}+\frac{I_{g}}{2}
\end{aligned}
$$

The standard deviation, in turn, is as follows:

$$
\begin{aligned}
\sigma_{I} & =\sqrt{\frac{1}{N}\left(\sum_{i=1}^{N / 2}\left(I_{g}+I_{p}-\langle I\rangle\right)^{2}+\sum_{i=1}^{N / 2}\left(I_{p}-\langle I\rangle\right)^{2}\right)} \\
& =\frac{I_{g}}{2}
\end{aligned}
$$

Thus, the standard deviation of the image batch yields the signal due only to the globar source $I_{g}$. Furthermore, the difference $\langle I\rangle-\sigma_{I}$ yields the signal due only to emission from the plasma $I_{p}$. Note that this analysis relies on the assumption that each image is collected over an interval much less than one half of the chopping period. If this assumption does not hold, then each image will contain only a partial fraction of the globar signal. For these experiments, a chopping frequency and sampling frequency of $150 \mathrm{~Hz}$ and $300 \mathrm{~Hz}$ were used, respectively. The camera integration time was $450 \mu \mathrm{s}$, much less than half the chopping period of $20 \mathrm{~ms}$. Thus the condition required for this analysis holds.

\section{Acknowledgments}

This work was supported under the ABLARADABLA program (ESA ITT A0/1-7987/14/NL/RA). Augustin Tibère-Inglesse is supported by CIFRE grand number 42701092/20160218/JSE with Airbus Safran Launchers. 


\section{References}

[1] John D. Anderson Jr. Hypersonic and High-Temperature Gas Dynamics. American Institute of Aeronautics and Astronautics, second edition, 2006.

[2] Mary G. D’Souza, Troy N. Eichmann, Daniel F. Potter, Richard G. Morgan, Timothy J. McIntyre, Peter A. Jacobs, and Neil R. Mudford. Observation of an ablating surface in expansion tunnel flow. AIAA Journal, 48(7):1557-1560, 2016/08/31 2010.

[3] Ricarda Wernitz, Christoph Eichhorn, Thomas Marynowski, and Georg Herdrich. Plasma wind tunnel investigation fo european ablators in nitrogen/methane using emission spectroscopy. International Journal of Spectroscopy, 2013(764321), 2013.

[4] Stefan Loehle, Tobias A. Hermann, Fabian Zander, Hannes Fulge, and Thomas Marynowski. Ablation Radiation Coupling Investigation in Earth Re-entry Using Plasma Wind Tunnel Experiments. American Institute of Aeronautics and Astronautics, 2016/08/31 2014.

[5] Bernd Helber, Olivier Chazot, Annick Hubin, and Thierry E. Magin. Microstructure and gas-surface interaction studies of a low-density carbon-bonded carbon fiber composite in atmospheric entry plasmas. Composites Part A: Applied Science and Manufacturing, 72:96 - 107, 2015.

[6] Tobias Hermann, Stefan Loehle, Pénélope Leyland, Lionel Marraffa, Jean-Marc Bouilly, and Stefanos Fasoulas. First results on ablation radiation coupling through optical emission spectroscopy from the vacuum ultraviolet to the visible. In European Symposium on Aerothermodynamics for Space Vehicles, 2015.

[7] Megan E. MacDonald, Carolyn M. Jacobs, Christophe O. Laux, Fabian Zander, and Richard G. Morgan. Measurements of air plasma/ablator interactions in an inductively coupled plasma torch. Journal of Thermophysics and Heat Transfer, 29(1):12-23, 2014.

[8] Michael Winter, Bradley Butler, Paul M. Danehy, Scott Splinter, Zhaojin Diao, Francesco Panerai, Alexandre Martin, and Sean Bailey. Characterization of Ablation Product Radiation Signatures of PICA and FiberForm. American Institute of Aeronautics and Astronautics, 2016/08/31 2016.

[9] Chul Park, Richard L. Jaffe, and Harry Partridge. Chemical-kinetic parameters of hyperbolic earth entry. Journal of Thermophysics and Heat Transfer, 15(1):76-90, 2016/08/31 2001.

[10] Alexandre Martin, Iain Boyd, Ioana Cozmuta, and Michael Wright. Chemistry Model for Ablating CarbonPhenolic Material During Atmospheric Re-Entry. American Institute of Aeronautics and Astronautics, 2016/09/01 2010.

[11] Christopher O. Johnston and Aaron M. Brandis. Features of afterbody radiative heating for earth entry. Journal of Spacecraft and Rockets, 52(1):105-119, 2016/07/26 2014.

[12] Christopher O. Johnston, Peter A. Gnoffo, and Alireza Mazaheri. Influence of coupled radiation and ablation on the aerothermodynamic environment of planetary entry vehicles. In Radiation and Gas-Surface Interaction Phenomena in High Speed Re-Entry, 2013.

[13] Boubert, P. and Vervisch, P. Laser-induced fluorescence measurements of $a^{3} \Pi$ metastable carbon monoxide in a high enthalpy flow. Eur. Phys. J. D, 17(1):43-48, 2001.

[14] Brett A. Cruden, Dinesh Prabhu, and Ramon Martinez. Absolute radiation measurement in Venus and Mars entry conditions. Journal of Spacecraft and Rockets, 49(6):1069-1079, 2016/08/31 2012.

[15] S. Depraz, M.Y. Perrin, and A. Soufiani. Infrared emission spectroscopy of $\mathrm{CO}_{2}$ at high temperature. part I: Experimental setup and source characterization. Journal of Quantitative Spectroscopy and Radiative Transfer, 113(1):1 - 13, 2012.

[16] Jean-Marc Bouilly. Asterm low density european ablative material: development status. In 8th European Workshop on Thermal Protection Systems \& Hot Structures, 2016.

[17] Megan MacDonald and Christophe O. Laux. Experimental characterization of ablation species in an air plasma ablating boundary layer. In 11th AIAA/ASME Joint Thermophysics and Heat Transfer Conference, number AIAA-2014-2251. American Institute of Aeronautics and Astronautics, June 2014.

[18] M. E. MacDonald, C. M. Jacobs, and C. O. Laux. Interaction of air plasma with ablating heat shield material. IEEE Transactions on Plasma Science, 42(10):2658-2659, Oct 2014.

[19] C O Laux, T G Spence, C H Kruger, and R N Zare. Optical diagnostics of atmospheric pressure air plasmas. 
Plasma Sources Science and Technology, 12(2):125, 2003.

[20] Christophe O. Laux. Optical Diagnostics and Radiative Emission of Air Plasmas. PhD thesis, Stanford University, Dept. of Mechanical Engineering, 1993.

[21] SPECAIR, Software Package, Ver. 3.0, SpectralFit S.A.S., http://www.spectralfit.com.

[22] Bernd Helber, Alessandro Turchi, James B. Scoggins, Annick Hubin, and Thierry E. Magin. Experimental investigation of ablation and pyrolysis processes of carbon-phenolic ablators in atmospheric entry plasmas. International Journal of Heat and Mass Transfer, 100:810 - 824, 2016.

[23] B. J. McBride and S. Gordon. Computer program for calculating and fitting thermodynamic functions. Technical report, NASA RP-1271, 1992. 